\title{
The Moon is a Special Place
}

A White Paper submitted to the Planetary Science Decadal Survey 2023-2032

Daniel P. Moriarty III

NASA GSFC / USRA

daniel.p.moriarty@nasa.gov 301-614-6843
Clive Neal

Notre Dame

cneal@nd.edu
Samuel Lawrence

NASA JSC

samuel.j.lawrence@nasa.gov

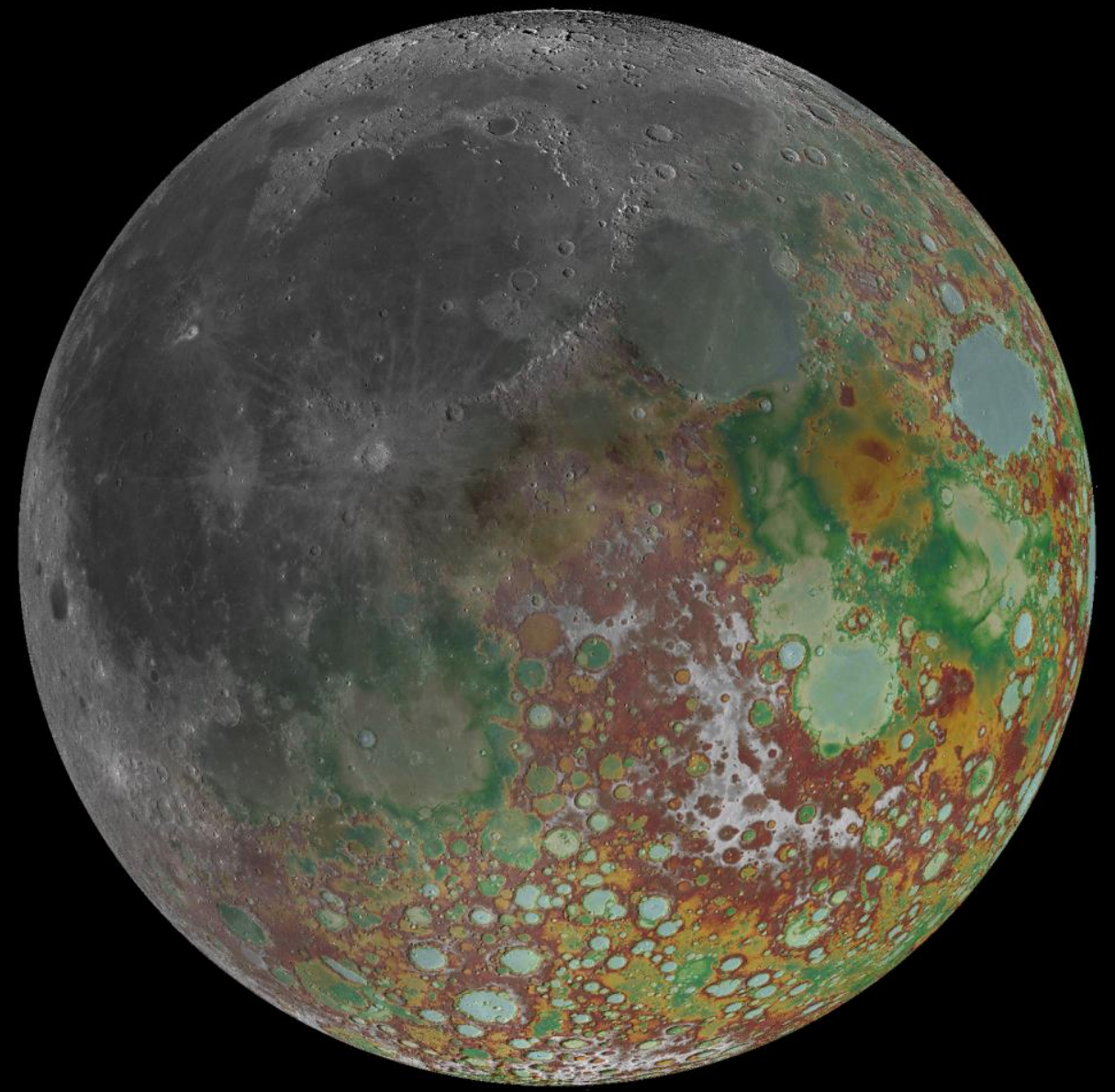

Signatories: Noah Petro (NASA GSFC), Sarah Valencia (NASA GSFC), Kelsey Young (NASA GSFC), James Tuttle Keane (Caltech), Heather Meyer (APL), Maria Banks (NASA GSFC), Ross Beyer (SETI), Deanna Phillips (UAH), Brett Denevi (APL), Heidi Haviland (NASA MSFC), Ryan Watkins (PSI), Jose Hurtado (UTEP), Shashwat Shukla (ITC), Rhushik Chandrachud (Mumbai University), Cass Runyon (CofC), Juliane Gross (Rutgers), Trevor Graff (NASA JSC), Stephen Elardo (UF), Sara Mazrouei (UToronto), Cesare Grava (SwRI), Wes Patterson (APL), Jennifer Whitten (Tulane), Erica Jawin (Smithsonian), Kirby Runyon (APL), Sriram Bhiravarasu (LPI), Nandita Kumari (Stony Brook), Thomas Magna (Czech Geological Survey), Nicholas Schmerr (UMD), Brad Jolliff (Washington University), Devanshu Jha (MVJCE, India), Kristen Bennett (USGS), Brian Day (ARC), Sean Gulick (UT), Jennifer Lopez (Astrobotic).

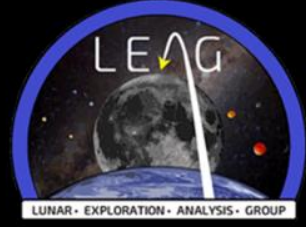


Key Recommendation: The Moon is scientifically rich, readily accessible, and harbors scientifically-interesting and exploration-enabling resources. For these reasons, the Moon provides a critical foundation for achieving fundamental planetary science and exploration goals. The Moon remains a target of the highest priority.

\section{Introduction}

The Moon is a special place. Shining bright in the night sky, the Moon is universal beacon of inspiration and connection. Throughout human history, the Moon has held immense cultural and intellectual importance across the globe. As our ambitions and abilities grow ever greater, a robust program of lunar exploration is critical in enabling our transition into a spacefaring civilization. Our closest planetary neighbor, it is by far the most accessible body in our Solar System. This unparalleled access facilitates a wide range of science and exploration goals. However, "convenience" is but a small part of what makes the Moon special. The Moon is a fascinating and unique body in its own right, and its study is central to numerous fundamental science questions.

This goal of this white paper is to outline and emphasize the special character of the Moon, and to advocate for its continued role as a high-priority science and exploration target in the Decadal Survey. In these seven pages, we will approach the special nature of the Moon through three perspectives: science, exploration, and society.

This paper draws from a collection of guiding documents generated by the lunar community. These are included in the reference list, and are an excellent resources providing deeper perspectives on the concepts briefly discussed here. Several of these important topics are discussed in greater detail in dedicated white papers also submitted to this survey.

\section{The Role of the Moon in Addressing Critical Science Goals}

As a species, unraveling the workings of the world around us is a perpetual yet rewarding challenge. Over the last several decades, significant developments in spaceflight and scientific capabilities have allowed us to expand our gaze from the planet beneath our feet, enabling the study of our planetary neighbors in remarkable detail. These scientific inquiries have shed light on the diverse processes shaping our Solar System, providing essential context for the understanding of our home planet.

The Apollo program was a touchstone of human scientific and engineering achievement, carrying the first humans to set foot on another world. Apollo surface experiments and returned samples profoundly shaped our understanding of planetary processes and the history of the solar system. In 2006, NASA commissioned the National Research Council to prepare for the next phase of lunar science and exploration through an assessment of the highest-priority science objectives. The Scientific Context for the Exploration of the Moon (NRC, 2007) succinctly delineates the Moon's unique role in addressing a diverse range of fundamental science questions. In 2017, the LEAG Advancing Science of the Moon Specific Action Team re-affirmed the relevance of the framework established by the $S C E M$ report towards ongoing lunar exploration efforts $(L E A G, 2018)$. Here, we review the eight concepts first described in the SCEM report emphasizing the Moon role as a cornerstone of planetary science, updated through the lens of several subsequent community documents and peer-reviewed publications (NACSC, 2007; NRC, 2011; Crawford et al., 2012; LEAG, 2016; LEAG, 2018; SSERVI, 2018).

- SCEM Concept 1: The bombardment history of the inner Solar System is uniquely revealed on the Moon. Understanding the flux of impactors through time is critically 


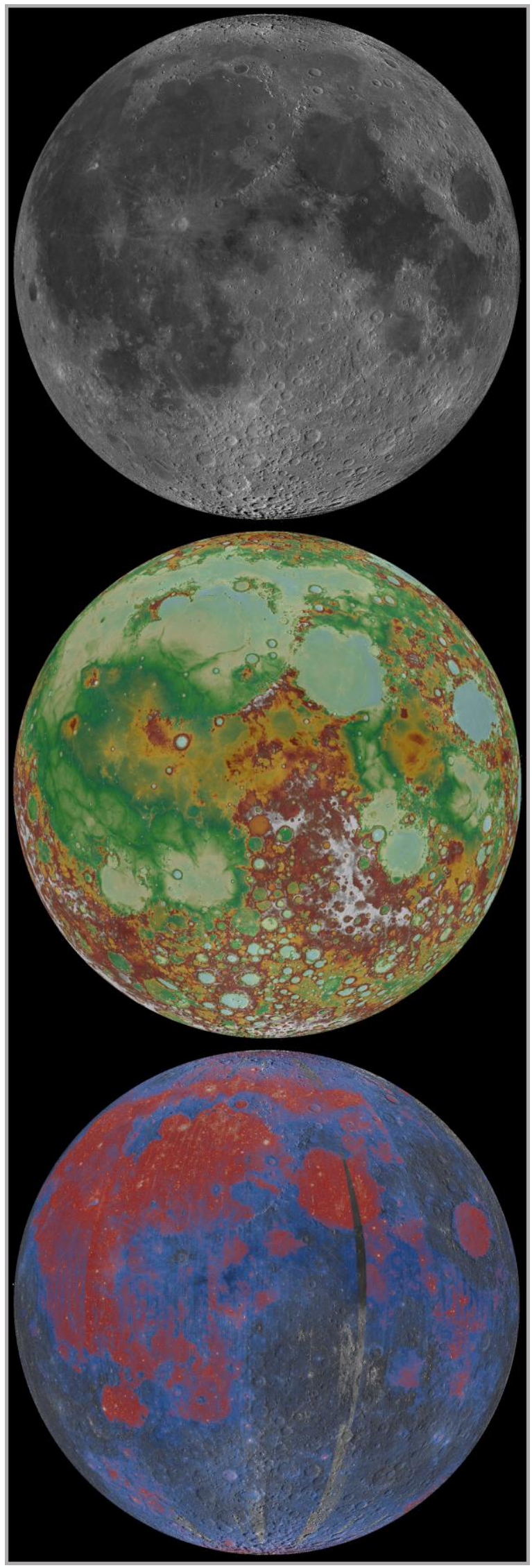

important for understanding the dynamical evolution of the Solar System, as well as the geological and biological implications of the correlated impact events. Because the Moon is a one-plate planet and undergoes only limited surface erosion, large-scale ancient structures such as impact basins are well-preserved on and near its surface. Basin formation is a highly energetic process that melts large volumes of target rock. This process resets isotope ratios in the resulting impact melt, functionally recording the timing of formation. Because these impact melts can be identified morphologically and compositionally from existing orbital data, lunar samples present a unique opportunity to unravel the bombardment history of the inner Solar System.

- SCEM Concept 2: The structure and composition of the lunar interior provide fundamental information on the evolution of a differentiated planetary body. The contemporary structure of the lunar crust and mantle is understood to have formed through crystallization of a global, deep melting event known as the "Lunar Magma Ocean." Magma oceans are thought to be a common feature of large terrestrial bodies, and are therefore a fundamental process driving formation and evolution of rocky planets across our Solar System and beyond. Because the Moon has not undergone plate tectonics, the resulting structure is well-preserved and can be determined through geophysical measurements. Relatedly, the dynamical evolution of the interior (particularly the core dynamo) is preserved in lunar magnetic anomalies. In addition to geophysical measurements, vast impact structures such as the South Pole - Aitken Basin serve as probes to the lunar interior, exposing

Figure 1: In LROC imagery (top), LOLA topography (middle), and $\mathrm{M}^{3}$ spectroscopy (bottom), the Moon exhibits great diversity in geologic features, geophysical structures, and composition. This diversity is the manifestation of the fundamental processes that shaped lunar formation and evolution. 
upper mantle materials at the surface. These represent some of the most accessible mantle materials in the Solar System and provide critical insight into the geochemical evolution of the lunar interior. Initial observations, models, and experiments demonstrate lunar evolution to be a complex process, even for a relatively straightforward and well-preserved case such as the Moon. If we hope to better understand these fundamental processes in a general sense, a "simple" case is an excellent starting point. Just as Galileo employed frictionless planes and massless strings to simplify mechanical concepts, the Moon serves as an idealized laboratory to study complex planetary evolution processes.

- SCEM Concept 3: Key planetary processes are manifested in the diversity of lunar crustal rocks. As mentioned above, products of the Lunar Magma Ocean including the primary crust and impact-excavated mantle are readily accessible at the lunar surface, revealing the Moon's early geochemical evolution. The Moon also exhibits a diverse range of secondary crustal products, discussed further in SCEM Concept 5. These lithologies provide insight into key planetary processes that shaped the Moon, with implications for similar processes on rocky planets across the Solar System and beyond. Global compositional information from orbital instruments such as the Moon Mineralogy Mapper reveal the compositional diversity of the Moon, guiding identification of the samples necessary to advance our understanding of these processes.

SCEM Concept 4: The lunar poles are special environments that bear witness to the volatile flux over the latter part of Solar System history. In particular, permanentlyshadowed regions at the lunar poles are among the coldest locations in the Solar System, in many cases exhibiting annual maximum temperatures below $110 \mathrm{~K}$. The extremely low temperature surfaces trap impinging volatile molecules, forming deposits that are stable over geologic timescales. Characterizing these volatile deposits will provide a historical record of the lunar volatile cycle, as well as the evolving flux and composition of the solar wind, interstellar volatiles, and larger volatile-bearing impactors (such as asteroids and comets). These impactors may have delivered significant quantities of organic materials to the lunar surface. The evolution of these compounds under harsh lunar surface conditions (radiation, impacts, thermal stresses, etc.) has implications for astrobiology.

SCEM Concept 5: Lunar volcanism provides a window into the thermal and compositional evolution of the Moon. The Moon exhibits a diverse range of magmatic emplacements reflecting the thermal evolution of the interior. These include widespread mare flood basalts, ancient Mg-suite plutons, volatile-driven pyroclastic deposits, anomalously young irregular mare patches, and a series of unusual silicic and other non-basaltic emplacements. The timing, morphology, and geochemistry of lunar volcanic events reflect the conditions of their formation, providing a benchmark to refine our understanding of volcanism and thermal evolution on the Moon and throughout the Solar System.

SCEM Concept 6: The Moon is an accessible laboratory for studying the impact process on planetary scales. From dust grain accretion to the formation of vast megabasins, impacts are the primary evolutionary driver of the Solar System and its planetary bodies. Large impacts cannot be replicated experimentally, and are not well-preserved on Earth. Therefore, we must turn to the extraterrestrial observational record to better understand these fundamental 
processes. In its rocks and structures, the Moon has preserved a record reflecting impacts over a wide range in scale, from micrometeorite pits to the $\sim 2500 \mathrm{~km}$ South Pole - Aitken Basin. The lunar record reveals the profound geophysical, morphological, stratigraphic, geochemical, and thermal, effects of impact processes at multiple scales.

SCEM Concept 7: The Moon is a natural laboratory for regolith processes and weathering on anhydrous airless bodies. Because the Moon is an airless body that lacks a strong magnetic field, its surface is subject to constant bombardment by micrometeorites, the solar wind, and other energetic particles. Additionally, the lack of an atmosphere contributes to an extreme diurnal temperature cycle, subjecting surface materials to thermal fatigue that contributes to regolith development. Understanding regolith formation and weathering processes is key for interpreting remote sensing data, unraveling sample provenance, and developing a sustained presence on the Moon and other planetary bodies. The lunar regolith may also preserve a record of solar and galactic events through capturing solar wind particles, interstellar dust particles, and recording the galactic cosmic ray flux.

SCEM Concept 8: Processes involved with the atmosphere and dust environment of the Moon are accessible for scientific study while the environment remains in a pristine state. Understanding the lunar volatile and dust cycle is an area of important fundamental science, and is critical for safeguarding and optimizing human and robotic lunar surface activities.

In 2017, the Lunar Exploration Analysis Group updated the list of eight driving science concepts from the SCEM report, having identified three additional high-priority science concepts which can be uniquely addressed through lunar analyses and exploration (LEAG, 2018):

- The lunar volatile cycle provides insight into the sources, sinks, mobility, and evolution of volatiles on airless bodies. Recent laboratory analyses have demonstrated that the lunar interior harbors more abundant volatiles than originally thought. Additionally, remote sensing data suggest diurnal variations in surface volatile abundance. These recent developments are among the highest-priority topics in lunar science, especially in the context of resuming human exploration of the Moon.

The origin of the Moon is intimately linked to the evolution of Earth, affecting the thermal states and subsequent geologic evolution of both bodies. The Moon is understood to have formed through a giant impact with the early Earth. This event would have profoundly shaped the evolution of our home planet, and its influence continues through tidal forces and other effects. Furthermore, the lunar regolith appears to contain samples of Earth's early crust and atmosphere, providing a historical record otherwise lost on Earth due to plate tectonics and other erosive processes.

Lunar tectonism and seismicity provide insight into the range of such processes on oneplate planets. The nature of moonquakes and the origin of tectonic features on the lunar surface is important to understand in the context of constraining the expression of diverse geophysical processes across the Solar System.

In addition to these planetary science concepts, the Moon serves as a distant, unique, and stable platform for conducting experiments and observations relevant to physics and astronomy 
(LEAG, 2016). For instance, the Moon has been used to refine our understanding general relativity (through laser ranging experiments), and could also be used to test quantum entanglement (through large baseline experiments). Additionally, the farside of the Moon is one of the most radio-quiet places in the solar system, as it is shielded from near-Earth interference and (partially from solar emissions. It is therefore an ideal location construct high-sensitivity radio telescopes to probe the early history of the universe (LEAG, 2016).

The above concepts represent a great depth and diversity of fundamental science questions. The Moon provides a unique opportunity to address these critical questions through characterization and exploration of a single, accessible planetary body. For these reasons, the Moon is an extremely high-priority science and exploration target.

\section{The Moon Uniquely Enables and Supports Solar System Exploration}

Exploration of the Moon is a richly-rewarding pursuit. Lunar exploration is a crucial first step towards further our species' spacefaring ambitions, enabling human and robotic exploration of Mars and beyond. A primary reason for this is the Moon's relative accessibility, in terms of both travel time/distance and the frequency of available launch periods. For example, travel to the Moon takes a few days, and launches can occur at any time throughout the year. Because of the large distances and orbital geometries, Mars is far less accessible. Minimum travel times are on the order of several months, and sensible launch periods are only available every two years.

For the Moon, this relative accessibility means that Earth-supplied technological innovation can be implemented on a timescale of weeks, not years. As an example, an issue with a regolith drill encountered during the Apollo 15 mission was rectified with a new drill design for the Apollo 16 mission months later. Because a similarly quick turnaround time would not be possible for Mars, it is important to develop and optimize technologies at the Moon before progressing to lessaccessible bodies.

However, "convenience" is far from the only reason to explore the Moon. Establishing a sustained presence on the lunar surface $(N A S A, 2020)$ is an essential and rewarding milestone on our journey to become a spacefaring species. Continued lunar exploration will develop and refine our approach to numerous enabling technologies and strategies required to (a) survive and operate under harsh lunar environmental conditions, (b) identify and utilize lunar resources such as metals, water, and other volatiles, and (c) establish and maintain a sustainable infrastructure satisfying power, communications, life support, and science requirements.

The lunar environment poses many hazards to both human and robotic surface activities. These hazards include a harsh radiation environment, lack of an atmosphere, low gravity, an extreme diurnal temperature cycle, and constant bombardment by micrometeorite impactors and mobile lunar dust grains. These conditions are not unique to the Moon, and may be encountered in exploration of planetary bodies throughout the Solar System. The Moon offers an excellent, accessible laboratory for characterizing these hazards to determine how they can be most effectively mitigated.

While the lunar environment poses several challenges to surface activities, other aspects of the Moon are enabling assets for exploration. In particular, permanently-shadowed regions at the lunar poles are thought to trap abundant volatiles, including water. These water deposits can be exploited for numerous purposes, including drinking water for astronauts and conversion to fuel for subsequent launches. Recent scientific analyses of lunar samples and orbital data indicate that lunar water is enhanced in certain surface materials (such as pyroclastic deposits) and is mobile over the diurnal cycle, providing potential non-polar sources of water. Metals and silicate 

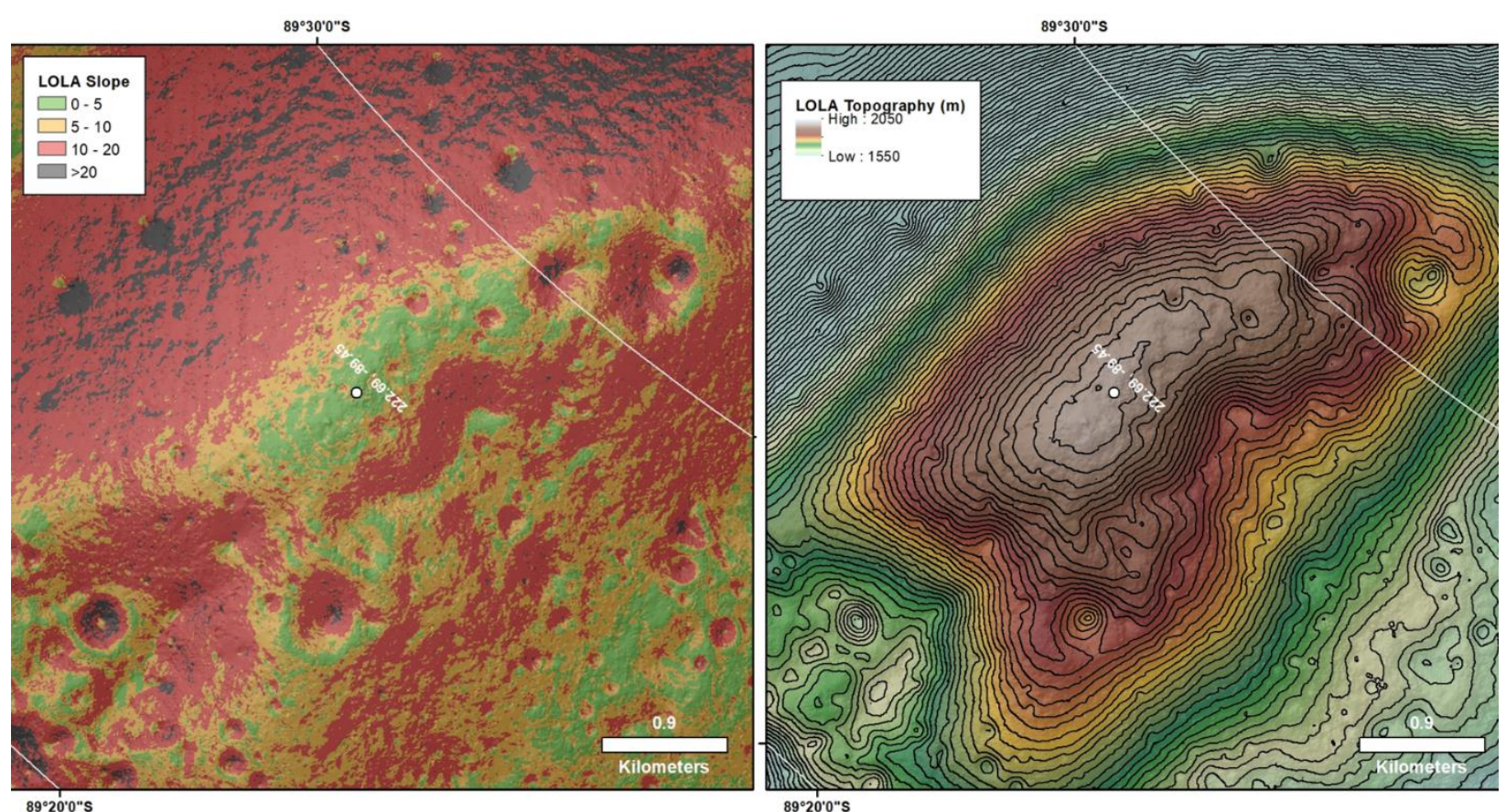

Figure 2: In preparation for the return of humans to the lunar surface via the Artemis program, NASA has evaluated several potential landing sites near the lunar south pole. The example site shown here, known as the "Shackleton - de Gerlache Connecting Ridge," is one of the most-illuminated locations on the lunar surface. From LOLA data, $5 \mathrm{~m} /$ pixel-resolution surface slope (left) and topography (right) maps capture the local landscape, revealing the locations of hazardous slopes as well as favorable terrain for conducting traverses and other surface activities.

materials distributed across the lunar surface may also provide significant exploration and/or economic value. Developing and optimizing strategies and tools to identify, harvest, and utilize these resources on the lunar surface is a critical step on our path toward becoming a spacefaring species, and enable detailed scientific investigations of such deposits as the data required for science and exploration are essentially the same (Lunar ISRU, 2019).

Through previous expeditions and decades of remote sensing observations, the surface of the Moon is uniquely well-understood. High-resolution imagery and topography facilitate landing site selection, hazard avoidance, infrastructure layout, and traverse routing. Transformative lunar and planetary science questions have been well-defined by the community, and the experiments and samples required are clearly stated. Compositional and other remote sensing data of the lunar surface reveal the distribution of these high-priority science samples, as well as resources enabling further exploration. The next era of lunar science and exploration is poised to be purposeful and impactful.

\section{The Unique Societal Impact of Lunar Science and Exploration}

As it shines beautifully in the night sky, the Moon has inspired millennia of artists, scholars, storytellers, adventurers, and others around the globe. As we elevate our gaze to our shared Moon, its exploration and study continue to elevate and inspire new generations of scientists and explorers. 
Recent lunar science and exploration efforts by agencies around the globe (including Europe, China, Japan, India, Canada, Israel, and Russia) indicate a burgeoning international interest and capability. Cooperation between international agencies has been formalized through the International Space Exploration Coordination Group, which seeks cooperation towards a world strategy for the exploration and utilization of the Moon. International interest in the Moon is further demonstrated by the success of meetings such as the European Lunar Symposium, which in 2020 featured $~ 500$ registrants from 36 countries. Study and exploration of our shared Moon has historically produced harmonious collaboration between the world's scientists and engineers - an ideal that world leaders should aspire to.

Recently, commercial entities have also demonstrated a growing interest in lunar pursuits. As an example, NASA is currently working with 14 companies to develop a Commercial Lunar Payload Service. This effort represents a shift from one-off, government-led missions to a more regular cadence of robotic lunar surface activity.

The appeal of the Moon is not limited to scientists and engineers. NASA develops and promotes numerous educational and outreach activities focused on the Moon. The public response to these programs has been overwhelmingly positive. For instance, NASA began an annual "International Observe the Moon Night" in 2010. The night brings together scientists and educators with members of the public to view and learn about our closest planetary neighbor. Over the past decade, this event has grown steadily. In 2019, over 2100 registered groups from 109 countries participated! Clearly, the Moon is a unique source of inspiration and wonder for curious minds around the world.

\section{Recommendations}

The Moon is a special place. Although we have learned much about the Moon over the past several decades of study and exploration, numerous high-priority science questions remain. Addressing these questions requires a robust program of lunar science and exploration, including orbital missions, robotic sample return, in situ analyses, human exploration, numerical modeling, laboratory experiments, terrestrial analog work, sample analyses, and data analysis. Furthermore, the long-term health of the lunar science community will be greatly strengthened through a clear commitment to diversity, inclusion, education, and public outreach.

\section{References:}

Crawford, I. A., Anand, M., Cockell, C. S., Falcke, H., Green, D. A., Jaumann, R., \& Wieczorek, M. A. (2012). Back to the Moon: The scientific rationale for resuming lunar surface exploration. Planetary and Space Science.

Lunar Exploration Analysis Group (LEAG) (2016) "The Lunar Exploration Roadmap: Exploring the Moon in the $21^{\text {st }}$ Century: Themes, Goals, Objectives, Investigations, and Priorities, 2016." Roadmap (LEAG 2016), Washington, DC.

Lunar Exploration Analysis Group (LEAG) (2018), "Advancing Science of the Moon: Report of the Specific Action Team." held 7-8 August 2017, Houston, TX.

Lunar In Situ Resource Utilization (ISRU) (2019), "Developing a New Space Economy through Lunar Resources and their Utilization." Columbia, MD.

NASA Advisory Council Science Committee (NACSC) (2007), “Workshop on Science Associated with the Lunar Exploration Architecture." Tempe, AZ.

NASA (2020), “NASA's Plan for Sustained Lunar Exploration and Development." Washington, DC.

National Research Council (NRC) (2007), "The Scientific Context for Exploration of the Moon." Washington, DC.

National Research Council (NRC) (2011), "Vision and Voyages for Planetary Science in the Decade 2013-2022",

National Academies Press, Washington, DC.

Solar System Exploration Virtual Institute (SSERVI) (2018), “Transformative Lunar Science.” Mountain View, CA. 